\title{
Neutrophil alkaline phosphatase in pregnancy
}

\author{
R. W. BEAL, W. M. F. READ AND P. A. TURVEY \\ From the Red Cross Blood Transfusion Service, Adelaide, South Australia
}

SYNOPSIS Neutrophil alkaline phosphatase levels have been studied in a group of 194 pregnant women, using a modified technique which permits more rapid counting of the required number of neutrophils with a degree of consistency comparable with standard methods.

No correlation was found between neutrophil alkaline phosphatase levels and the period of gestation; in contrast to previous findings, no differences were observed between the means for tests done in early pregnancy and those performed later in pregnancy. There was no demonstrable relationship between neutrophil alkaline phosphatase levels and age, parity, and white cell count. Elevated levels were also observed in women taking oral contraceptives. Thus, although the neutrophil alkaline phosphatase has diagnostic value in certain other disease states, the above findings suggest that it is of little or no value in pregnancy.

The possibility of hormonal control of the elevated level of neutrophil alkaline phosphatase seen in pregnancy and simulated pregnancy is discussed, as is the possible relationship to increases in serum alkaline phosphatase in pregnancy.

The pregnant state is one of a number of clinical situations in which an elevation of the neutrophil alkaline phosphatase has been demonstrated. Since the first descriptions of this phenomenon in pregnancy by Valentine and others (Valentine et al., 1952; Valentine and Beck, 1957; Pritchard, 1957), several attempts have been made to utilize the neutrophil akaline phosphatase test as a confirmatory investigation in early pregnancy (Quigley, Dawson, Hyun, and Custer, 1960; Harer and Quigley, 1961; Chang, 1963). It has also been suggested that the level of neutrophil alkaline phosphatase rises during pregnancy (Quigley et al., 1960; Rutenburg, Rosales, and Bennett, 1965). The present study has shown that the level is not related to the duration of the pregnancy, nor to age, parity, the total white cell count, or the neutrophil count. Further, we have confirmed the recent observations of Gordon and Hunter (1965) that the simulation of the pregnant state produced by oral contraceptives is accompanied by high neutrophil alkaline phosphatase levels, thus rendering this test of less value than was previously thought.

A modification of the standard technique has been used in order to reduce the time required for scoring slides, without decreasing accuracy or efficiency.

Received for publication 8 March 1967.

\section{MATERIALS AND METHODS}

PREGNANT WOMEN The 205 pregnant women investigated were chosen at random from those attending the reference laboratory of this Service for routine antenatal serological testing. Information was available in most instances concerning age, parity, and estimated date of confinement.

NORMAL CONTROLS The normal controls were mostly unmarried female laboratory staff, but blood from normal male and female blood donors was also used to establish the normal range.

TECHNIQUE Blood from the individuals studied was collected by venepuncture into sterile containers, using sequestrene as the anticoagulant. Thin films were made immediately and air-dried. The method used was basically that described by Hayhoe and Quaglino (1960), which is itself a modification of the cytochemical technique of Kaplow (1955). Methyl green (2\% w/v) was used as the counterstain.

Blood from which concentrates were prepared was collected into a dextran-citrate mixture containing 1 volume of $6 \%$ dextran and 1 volume of $3.8 \%$ sodium citrate for each 10 volumes of blood. After sedimentation, the supernatant was centrifuged for 10 minutes at 2,000 r.p.m., and slides were prepared from the cell-rich layer at the bottom of the centrifuge tube.

A standardized scoring system was used, as suggested by Dacie and Lewis (1963), except that cells which showed 
'barely visible diffuse staining' without granules were scored as $\mathbf{0}$; thus our normal range is considerably lower than some previously reported. White cell counts and differential counts were done by standard methods (Dacie and Lewis, 1963).

The figures quoted for neutrophil alkaline phosphatase scores in this paper are the means of at least three readings, done by at least two observers on a minimum of two separate slides stained simultaneously. Reasonable agreement was obtained by the observers, who scored the slides without knowledge of the scores obtained by the other. The differential counts recorded are the means of at least one differential count by each observer.

\section{RESULTS}

NORMALS Females taking oral contraceptives were excluded from the group of normal controls, as levels of more than three times the upper limit of normal were observed at certain times during the menstrual cycle in some of the women studied.

The range of scores for neutrophil alkaline phosphatase obtained on normal controls was between 5 and 25; no difference was observed between the readings obtained in males and in females other than those specified above.

PREGNANT WOMEN The range of scores for neutrophil alkaline phosphatase obtained in 194 pregnant women was between 28 and 145. One woman was studied twice during the same pregnancy (the results being similar), and 10 were excluded on technical grounds, that is, insufficient results were obtained, or because there were doubts concerning whether or not the woman was pregnant.

RElationship To STAGE OF PREgnANCY Table I shows the scores related to the periods of gestation. There is no significant difference between the scores

TABLE I

NEUTROPHIL ALKALINE PHOSPHATASE SCORES (N.A.P.) AND STAGE OF PREGNANCY

\begin{tabular}{lcl}
$\begin{array}{l}\text { Weeks of } \\
\text { Gestation }\end{array}$ & $\begin{array}{c}\text { No. in } \\
\text { Group }\end{array}$ & $\begin{array}{l}\text { Mean N.A.P. } \\
\text { Score }\end{array}$ \\
\hline $0-8$ & 5 & 55 \\
$9-12$ & 30 & 65 \\
$13-16$ & 28 & 69 \\
$17-20$ & 22 & 73 \\
$21-24$ & 13 & 84 \\
$25-28$ & 15 & 79 \\
$29-32$ & 13 & 64 \\
$33-36$ & 45 & 74 \\
$37-40$ & 20 & 84 \\
& $191^{1}$ & \\
& Overall mean $=73$ & \\
& $\chi^{2}=10 \cdot 46$ &
\end{tabular}

${ }^{1}$ Data concerning E.D.C. not available for 3 women. for the successive four-week stages of pregnancy. No differences were demonstrable when these figures $\stackrel{?}{\rightarrow}$ were regrouped into three larger categories, $0-160$ weeks, 17-28 weeks, and 29-40 weeks $\left(\chi^{2}=1.091\right.$; $0 \cdot 70>\mathbf{P}>0 \cdot 50$ ).

RELATIONSHIP TO PARITY The results obtained have been considered in relationship to the parity of the $-\vec{A}$ subjects studied; the results are set out in Table II. There is no significant variation of neutrophil alkaline $\vec{\omega}$ phosphatase with parity. There is also no differences for the means of the scores for women during their용 first pregnancy and those in their second or subsequent if pregnancy.

TABLE II

NEUTROPHIL ALKALINE PHOSPHATASE (N.A.P.) SCORES AND PARITY

\begin{tabular}{lcl}
$\begin{array}{l}\text { No. of Previous } \\
\text { Pregnancies }\end{array}$ & $\begin{array}{l}\text { No. in } \\
\text { Group }\end{array}$ & $\begin{array}{l}\text { Mean N.A.P. } \\
\text { Score }\end{array}$ \\
\hline 0 & 66 & 72 \\
1 & 47 & 71 \\
2 & 34 & 79 \\
3 & 19 & 69 \\
4 & 10 & 64 \\
5 or more & 14 & 74 \\
& $190^{1}$ & \\
& Overall mean $=73$ & \\
& $\chi^{2}=1.86$ & \\
& D.F. $=5$ &
\end{tabular}

${ }^{1}$ Details of parity not available for 4 women.

RELATIONSHIP TO AGE The results obtained have $\underset{7}{\overrightarrow{2}}$ been considered in relationship to the age of the subjects studied (Table III); there is no significant $=$ alteration in scores for neutrophil alkaline phos-o phatase with variation in age.

\section{TABLE III}

NEUTROPHIL ALKALINE PHOSPHATASE (N.A.P.) SCORES AND AGE

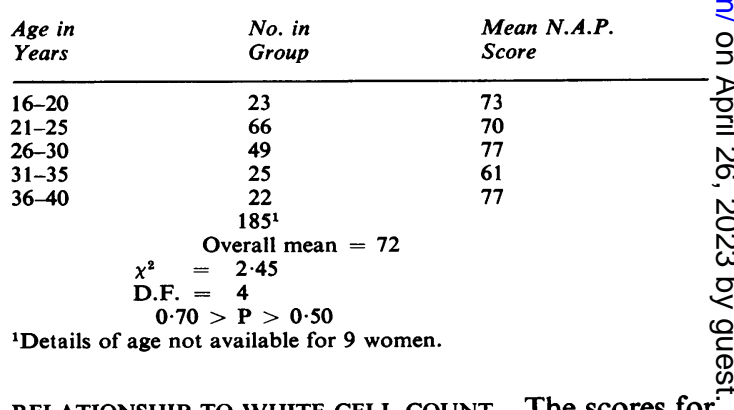

RELATIONSHIP TO WHITE CELL COUNT The scores for neutrophil alkaline phosphatase were plotted against the white cell counts obtained in the 31 women inल whom this correlation was sought. There was no $\frac{?}{\mathrm{P}}$ demonstrable relationship existing between these 
scores and the white cell counts, nor between the number of neutrophils present (calculated from the differential counts) and the neutrophil alkaline phosphatase score. The only exception is that if the total neutrophil count was greater than 7,000 per c.mm., the score for neutrophil alkaline phosphatase was consistently above 100 in those studied; this may have an association with infection in pregnancy.

COMPARISON OF METHODS Paired slides were prepared from blood obtained from 73 women in the series; one slide was prepared and stained in the conventional manner, and the other was prepared from the concentrated white cell suspension. The mean neutrophil alkaline phosphatase score for the series prepared normally was 86 , and the mean score for the slides prepared from the concentrates was 85 . The mean of the differences between each of the 73 pairs was 11 .

\section{DISCUSSION}

One disadvantage of the standard histochemical techniques for determination of neutrophil alkaline phosphatase has been the length of time required to count the requisite number of cells, resulting in observer fatigue and possible inaccuracy or inconsistency in scoring. The modification used for this study enables the time spent in examining the slide to be reduced to a quarter of that required under standard conditions. The use of cell concentrates enables observer efficiency to be maintained for longer periods, and as a result more slides may be scored in the same period of time. The proximity of stained neutrophils to each other within the same microscope field which results from the concentrated preparation is also advantageous in achieving consistency of scoring. A comparison of this technique with the standard method shows it to possess a comparable degree of accuracy within the limitation of the method per se.

The neutrophil alkaline phosphatase score has been put forward as a possible confirmatory test for pregnancy; it is said to be raised early in pregnancy (Quigley et al., 1960; In Whan Chang, 1963) and levels of neutrophil alkaline phosphatase are said to rise as the pregnancy progresses. However, the results of this study show that an elevated neutrophil alkaline phosphatase score is as common a finding early in pregnancy as later on; there is no significant difference demonstrable between levels obtained in early pregnancy and those obtained in late pregnancy.

Furthermore, the demonstration of elevated neutrophil alkaline phosphatase scores in women who are in a state of simulated pregnancy as a result of taking oral contraceptives renders the neutrophil alkaline phosphatase test valueless as a diagnostic aid in pregnancy.

These findings revive the suggestion that the abnormal metabolism of neutrophil alkaline phosphatase in pregnancy has a hormonal basis (Valentine, Follette, Solomon, and Reynolds, 1957; In Whan Chang, 1963). No pattern is apparent from these present results which could be explained by the variations in hormone levels which occur during normal pregnancy.

Gordon and Hunter, (1965) have demonstrated cyclical variations in neutrophil alkaline phosphatase activity in normal premenopausal women, and have observed irregular elevations of the enzyme in women taking oral contraceptives. They were able to locate only one previous reference to raised neutrophil alkaline phosphatase levels in relation to menstruation (Borel, Frei, and Vannotti, 1958). Gordon and Hunter also suggest that the control of neutrophil alkaline phosphatase metabolism may be under either ovarian or pituitary control.

It is now well recognized that serum alkaline phosphatase levels are elevated in pregnancy (Kitchener, Neale, Posen, and Brudenell-Woods, 1965), possibly because of the presence of quantities of placental alkaline phosphatase (Birkett, Done, Neale, and Posen, 1966). It is possible that the application of recently developed techniques for the demonstration of neutrophil alkaline phosphatase isoenzymes (Robinson, Pierce, and Goldstein, 1965; Robinson, Pierce, Goldstein, and Rosse, 1966) may reveal a similar basis for the increases seen in pregnancy, and possibly for those seen in simulated pregnancy.

\section{REFERENCES}

Birkett. D. J., Done, J., Neale, F. C., and Posen, S. (1966). Brit. med. J., 1. 1210.

Bore!, C., Frei, J., and Vannotti, A. (1958). Helv. physiol. pharmacol. Acta, 16, c53. Cited by Gordon \& Hunter (1965).

Chang, I. W. (1963). Amer. J. Obstet. Gynec., 86, 903.

Dacie, J. V., and Lewis, S. M. (1963). Practical Haematology, Churchill, London.

Gordon, S., and Hunter, E. (1965). Med. J. Aust., 2, 13.

Harer, W. B., Jr., and Quigley, H. J. (1961). Obstet. and Gynec., 17, 238.

Hayhoe, F. G. J., and Quaglino, D. (1960). Brit. J. Haemat., 6, 381.

Kaplow, L. S. (1955). Blood, 10, 1023.

Kitchener, P. N., Neale, F. C., Posen, S., and Brudenell-Woods, J. (1965). Amer. J. clin. Path., 44, 654.

Pritchard, J. A. (1957). J. Lab. clin. Med., 50, 432.

Quigley, H. J., Dawson, E. A., Hyun, B. H., and Custer, R. P. (1960). Amer. J. clin, Path., 33, 109.

Robinson, J. C., Pierce, J. E., and Goldstein, D. P. (1965). Science, $150,58$.

,,--- , and Rosse, W. R. (1966). Lancet, 2, 805.

Rutenburg, A. M., Rosales, C. L., and Bennett, J. M. (1965). J. Lab. clin. Med., 65, 698.

Valentine, W. N., and Beck, W. S. (1951). Ibid., 38, 39.

-, Follette, J. H., Solomon, D. H., and Reynolds, J. (1957). Ibid., 49, 723. 